\title{
Can ambulance personnel intubate?
}

\author{
A. J. GRAY, D. CARTLIDGE \& M. C. GAVALAS \\ Accident and Emergency Department, Stockport Infirmary, Stockport, Cheshire
}

\section{SUMMARY}

To assess the performance of intubation skills by advanced trained ambulance personnel, a prospective study was carried out of the intubation of cardiac arrest victims by ambulance personnel in the field.

Twenty-eight ambulance personnel attempted the intubation of 87 patients over 2 years. Eighty-five patients were initially successfully intubated, one endotracheal tube became displaced and cuff leakage occurred on three occasions. This was treated appropriately in each instance.

Ambulance personnel can perform the skills of intubation successfully in out-ofhospital cardiac arrest victims.

\section{INTRODUCTION}

In 1984 ambulance personnel in Stockport were trained in the skills of defibrillation (Gray et al., 1987). It was decided subsequently that selected personnel should be trained in intravenous cannulation and endotracheal intubation, for established cardio-respiratory arrest. Patients in ventricular fibrillation were intubated after three unsuccessful defibrillation attempts and patients in asystole were intubated after a period of basic life support.

The aim of the study was not to assess the effectiveness of paramedic resuscitation but to assess prospectively whether ambulance personnel could intubate patients rapidly and safely.

\section{METHODS}

The ambulance personnel chosen received $4 \mathrm{~h}$ of lectures based on the National Health Service Training Authority syllabus. The practical skills of intubation were

Correspondence: A. J. Gray, Accident \& Emergency Department, Stockport Infirmary, Stockport, Cheshire SK1 3UJ. 
learned in the operating theatre under the supervision of a consultant anaesthetist. Fifteen successful intubations were required in theatre, five on cadavers and five on mannikins. The candidates sat an examination of 20 short note questions and their intubation skills were examined on two mannikins which they were required to intubate within $1 \mathrm{~min}$ each. This assessment was performed by independent Consultant Anaesthetists from a teaching hospital in another district.

The successful candidates were allowed to intubate cardiac arrest victims following the agreed protocol (Appendix 1). After each attempt at intubation success and complications were assessed and recorded by the receiving doctor in the resuscitation room. A proforma (Appendix 2) was completed by the ambulance personnel before they left the resuscitation room.

\section{RESULTS}

Twenty-eight ambulance personnel were trained. Intubation was attempted on $\stackrel{9}{\rightarrow}$ 87 patients over a period of 2 years. Sixty-five $(75 \%)$ patients required the use of suction during intubation to clear mucous or vomit from the airway. In 62 (71\%) patients the intubation procedure was described as 'easy', the remaining 25 were classed as 'difficult'; this was purely a subjective assessment by the ambulance personnel themselves.

Eighty-five patients were intubated, although only 81 patients remained intu bated at the time they entered the resuscitation room. None of these patients? when examined by the receiving doctor, were found to have the endotracheal tube in the oesophagus.

In three patients the cuff on the endotracheal tube was leaking or had burst. This was recognized on all three occasions. The endotracheal tube was removed and ventilation maintained with a bag and mask technique using an orotracheal airway. The endotracheal tube became displaced in one patient necessitating its removal. Once again ventilation was maintained with a bag and mask. The use of Magill's forceps was required for the intubation of one patient.

In two patients $(2 \%)$, the ambulance personnel could not visualize the vocal cords and the intubation attempt was abandoned as per the protocol. These patients were maintained with an oro-pharyngeal airway and bag and mask ventilation during their transfer to the A\&E department.

In one case the protocol was not adhered to when the attempt at intubation lasted $1.5 \mathrm{~min}$.

\section{DISCUSSION}

Endotracheal intubation is the definitive method of airway management in the completely unresponsive or arrested patient.

It may also be desirable in cases of acute respiratory failure from other causes 
and early control of the airway in major trauma victims is of proven value (Spence et al., 1988).

The benefits of endotracheal intubation in the pre-hospital environment are unproven although 38 of 65 survivors of cardiac arrest resuscitated by ambulance personnel in West Yorkshire were intubated. The influence of intubation on their survival was not assessed. Endotracheal intubation by non-anaesthetists is controversial. Scott has pointed out the dangers of prolonged intubation attempts and incorrect tube placement by the inadequately trained, suggesting that those not trained in anaesthesia are best advised to use mouth to mouth breathing only (Scott, 1986). We do not agree with this advice; to do so, would deny the severely compromised patient an optimum oxygenation and protection from aspiration that endotracheal intubation affords.

Paramedic training of U.K. Ambulance Personnel is now widely established and is regulated under guidelines from the NHS training authority. In an audit from 1988 Extended Aid was practised in 47/88 regions and 4929 intubations had been performed (Vincent, 1990). The benefits of pre-hospital defibrillation have been established but the value of, and success of, other interventions such as intubation and drug administration is less easy to calculate. The results of this study have shown that ambulance personnel trained under a local initiative can intubate cardiac arrest victims safely with a low incidence of complications and according to an agreed protocol. The overall success rate was $98 \%$ with a complication rate of $6 \%$. In the U.S.A., Stewart described an overall success of $94.5 \%$ once field experience was established (Stewart et al., 1985) and Jacobs $96.6 \%$ with no complications (Jacobs et al., 1983). This study was confined to non-trauma patients who had suffered cardiac arrest. We would therefore expect our results to be better than those series which included trauma patients in whom increased difficulty could be expected due to the presence of injury adjacent to the airway and the constraints imposed by a potentially injured cervical spine. Our protocol was also less rigorous perhaps allowing $1 \mathrm{~min}$ for intubation compared with $45 \mathrm{~s}$ and $15 \mathrm{~s}$ respectively. We are not aware of any time guidelines for intubation in the present ambulance staff training committee literature.

We are concerned that the endotracheal tube cuff burst on three occasions. This is a far higher incidence than occurs in normal anaesthetic practice and we cannot be sure that this did not represent over inflation causing bursting of the cuff of an incorrectly placed tube: even if this had been the case, the overall success rate would still have been $94 \%$.

Intubation of the right main stem bronchus is a potential complication. In this series, tube position was confirmed by auscultation of the lung fields by the receiving doctor. The majority of these patients had already died and their position was not confirmed by X-ray.

The indications for ambulance personnel intubation in the field are controversial and remain undefined. However, if this technique can be achieved expeditiously and with a low incidence of complications, the advantages of using this method of ensuring oxygenation and protection against aspiration during initial resuscitation and transfer may well outweigh the delay of a few seconds whilst it is instituted. We have demonstrated that this difficult skill can be performed successfully in the 
field by ambulance personnel; further research is required urgently to determine its effect.

\section{ACKNOWLEDGEMENTS}

We would like to thank Dr P. Nightingale and Dr A. Gilman for their help in this study.

\section{REFERENCES}

Gray A. J., Redmond A. D. \& Martin M. (1987) Use of the automatic external defibrillator Pacemaker by $\underset{\perp}{ \pm}$ ambulance personnel. The Stockport experience. British Medical Journal 294, 1133-1135.

Jacobs L. M., Berrizbeitia, L. D., Bennet B. \& Madigan C. Endotracheal intubation in the prehospital phase of emergency medical care. Journal of the American Medical Association 250(16), 2175-2177.

Scott D. B. (1986) Endotrachael intubation; friend or foe. British Medical Journal 292, 157-158.

Spence H. T., Redmond A. D. \& Edwards J. D. (1988) Trauma Audit - the use of TRISS. Health Trends 20, 94-97.

Stewart R. D., Paris P. M., Winter P. M., Pelton G. H. \& Cannon G. M. (1985) Field endotracheal intubation by paramedical personnel. Chest 3, 341-345.

Vincent R. (1990) In: ABC of Resuscitation, 2nd Ed (Ed. Evans T. R':) British Medical Journal, London? !

Wright D., Bannister J., Ryder M. \& Mackintosh A. F. (1990) Resuscitation of patients with cardiac arresi. by ambulance staff with extended training in West Yorkshire. British Medical Journal 301, 602-603..

\section{APPENDIX 1}

Protocol for the intubation and defibrillation of cardiac arrest patients by ambulance personnel

(1) Establish a history of sudden collapse.

(2) Approach the patient and enquire in a loud voice if they are alright.

(3) If there is no response shake the patient vigorously and again enquire if they are alright.

(4) If there is no response, turn the patient flat on their back and with two fingers actively remove any foreign bodies, vomit or false teeth and then grip the tongue and pull it forward. If there is no response quickly confirm that there is no carotid pulse. If not, give two breaths into the patient's mouth. Strike them firmly once in the middle of the breastbone.

(5) If there is no response then commence closed cardiac compression. With the patient lying flat on their back on a firm surface, place the heel of one hand firmly in the middle of the chest and the heel of the other hand over it. In a rocking motion use the weight of your body transmitted through your extended elbows to compress the chest of an adult for about two inches. This motion $N$ need not be very fast and should be of a "squeezing-squashing" motion. Do this five times. If $N$ there is no response then give two breaths into the patients mouth.

(6) If there is still no response, conncet the patient to the defibrillator. Turn on the machine and ensure that nobody is touching the patient and that all passers-by are well clear.

(7) If the patient is in ventricular fibrillation then administer three shocks. If the patient does not recover then switch off the machine and prepare to intubate.

(8) Position the patient by extension at the occipital joint. You can achieve this by packing a small pad under the patient's head.

(9) Apply firm pressure at the back of the head. This makes the mouth fall open. 
(10) Introduce the blade of the laryngoscope to the right of the midline. Retract the tongue to the left.

(11) Follow the landmarks of the roof of the mouth, uvula and the tip of the epiglottis.

(12) Position the tip of the blade anterior to epiglottis and gently lift the epiglottis upwards. This reveals the larynx and vocal cords. Do not lever the laryngoscope on the teeth.

(13) Pass the lubricated endotracheal tube, under direct vision, through the Rima glottis inlet into the trachea.

(14) Insert until the connector is just outside the upper lip.

(15) Connect the catheter mount and the inflating bag and ventilate the lungs. At this stage most of the inflated air escapes around the tube and you hear a characteristic sound.

(16) Inflate the cuff until no air leaks are heard.

(17) Clamp off the pilot tubing using the artery forceps.

\section{Points}

(i) The endotracheal tube must be seen to pass through the cords.

(ii) After intubating check that chest expansion is equal and that there are breath sounds in both lungs. If these requirements are not achieved the tube should be withdrawn and the patient managed by bag and mask ventilation with an oropharyngeal airway as before.

The intubation sequence should not take longer than $1 \mathrm{~min}$.

\section{APPENDIX 2}

To be completed by all ambulance personnel on all patients intubated out-of-hospital

Patient's name

Address

Ambulance personnel names

Date

Defibrillator used - YES/NO

Tube size $-9.5 / 8.5$

Intubation - Easy

Difficult

Failed

Suction - Yes/No

Approximate time taken for intubation

Any problems? 